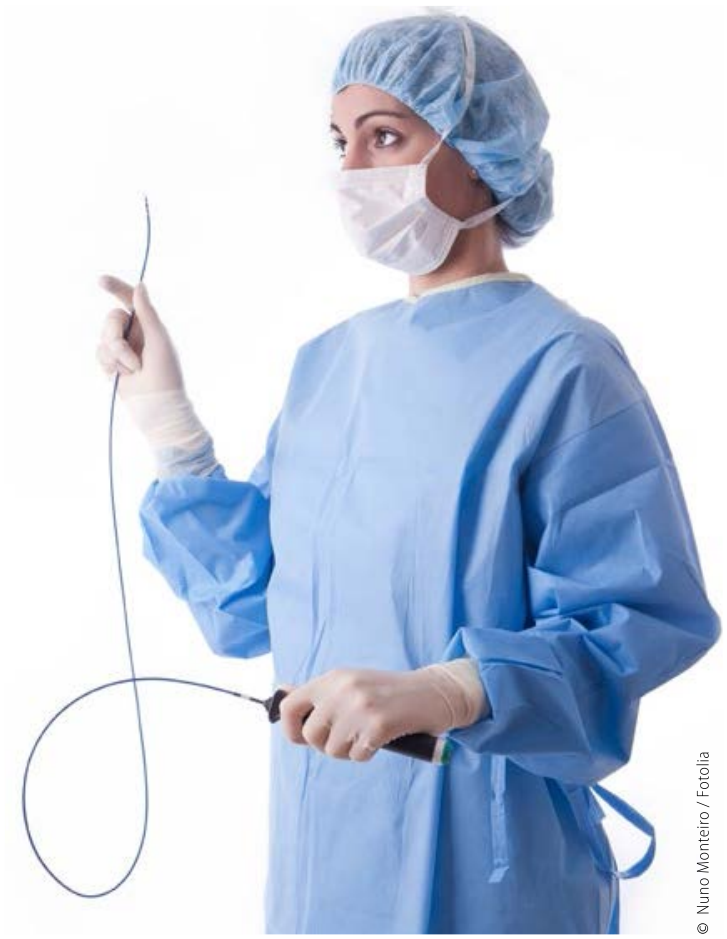

Die Anzahl der Linksherzkatheter-Untersuchungen und $\mathrm{PCl}$ sind gegenüber dem Vorjahr gestiegen.

\section{DATEN UND FAKTEN}

\subsection{7}

perkutane Koronarinterventionen ( $\mathrm{PCI}$ ) sind im Jahr 2014 in Deutschland durchgeführt worden. Diese auf einer Hochrechnung gründende Zahl nennt der Anfang des Jahres vorgestellte „Deutsche Herzbericht 2015". Gegenüber dem Vorjahr ist die PCl-Zahl damit um $5,4 \%$ gestiegen. Die Gesamtzahl aller Linksherzkatheter-Untersuchungen wird mit 906 843 beziffert. Auch hier gab es gegenüber 2013 eine Zunahme um 2,4\%

\section{INFOS IM INTERNET}

Alle Folgen der Sommer-Akademie finden Sie im Web unter www.aerztezeitung.de

Auf www.kardiologie.org finden Sie ein reiches Angebot an Informationen aus dem Bereich Kardiologie, darunter aktuelle Kongressberichte. Das Onlineportal ist ein gemeinsames Projekt der Deutschen Gesellschaft für Kardiologie (DGK), des Bundesverbandes Niedergelassener Kardiologen (BNK) e.V. und Springer Medizin.

www.escardio.org bietet als Website der Europäischen Gesellschaft für Kardiologie (ESC) Zugang zu den europäischen Leitlinien im Bereich Kardiologie, darunter auch die 2016 aktualisierten Herzinsuffizienz-Leitlinien.

\title{
ARNI-Therapie ermöglicht längeres Leben
}

Grundlage für die Aufnahme von Sacubitril/Valsartan in die neuen Herzinsuffizienz-Leitlinien bilden die Daten der Studie PARADIGM HF.

Sacubitril/Valsartan (Entresto ${ }^{\circ}$ ) ist seit November 2015 für die Indikation symptomatische chronische Herzinsuffizienz mit reduzierter Ejektionsfraktion (HFrEF) zugelassen. Das neue Arzneimittel enthält in einer Tablette den $\mathrm{AT}_{1}$-Rezeptorblocker Valsartan und den Neprilysin-Hemmer Sacubitril, es wird deshalb auch als ARNI (Angiotensin-Rezeptor/ Neprilysin-Inhibitor) bezeichnet. Durch Hemmung von Neprilysin wird der Abbau natriuretischer Peptide verhindert und so deren kardioprotektive Wirkung verstärkt, zudem wird das bei Herzinsuffizienz überaktive Renin-Angiotensin-Aldosteron-System supprimiert.

Den klinischen Nutzen dieser neuen Therapie dokumentieren die Ergebnisse der Studie PARADIGM-HF. PARADIGM-HF ist mit 8442 Patienten die bis dato größte Studie, in der je ein neues Arzneimittel bei Herzinsuffizienz klinisch geprüft worden ist. Vergleichssub- stanz war nicht wie üblich Placebo, sondern der ACE-Hemmer Enalapril, dessen prognostisch günstige Wirkung bei Herzinsuffizienz durch placebokontrollierte Studien belegt ist.

\section{Gesamtsterberate gesenkt}

Sacubitril/Valsartan war in puncto klinische Wirksamkeit dem ACE-Hemmer klar überlegen. Kardiovaskuläre Todesfälle und Klinikeinweisungen wegen Herzinsuffizienz (primärer kombinierter Endpunkt) wurden im Vergleich signifikant um 20\% reduziert. Die Gesamtsterberate war signifikant um $16 \%$ und die Rate für die kardiovaskuläre Mortalität signifikant um 20\% niedriger. Die Zahl der Klinikeinweisungen wegen sich verschlechternder Herzinsuffizienz wurde signifikant um $21 \%$ verringert.

Eine Gruppe von US-Untersuchern hat auf Basis dieser Ergebnisse jüngst versucht, den Überlebensvorteil bei adäquater Implementierung von Sacubitril/Valsartan in den Praxisalltag zu quantifizieren. Nach ihrer Schätzung ließen sich durch diese Therapie allein in den USA pro Jahr fast 30.000 Todesfälle verhindern. (ob)

\section{Vorhofflimmern}

\section{Praxisleitfaden zu DOAK aktualisiert}

Die European Heart Rhythm Association (EHRA) hat 2013 erstmals einen Praxisleitfaden (practical guide) zur Behandlung mit nicht über Vitamin-K-Antagonisierung wirksamen Gerinnungshemmern (Thrombinhemmer, Faktor-Xa-Hemmer, auch als NOAK oder DOAK bezeichnet) bei Vorhofflimmern herausgegeben. Die PocketVersion stieß mit weltweit mehr als 350.000 vertriebenen Exemplaren nicht nur bei Kardiologen auf sehr großes Interesse.

\section{Ursprüngliches Konzept beibehalten} Seit Mitte Juni gibt es nun eine auf der Grundlage neuer Studiendaten aktualisierte Version dieses Leitfadens. Das ur- sprüngliche Konzept, die praktische Anwendung von NOAKs in 15 klinischen Szenarien darzustellen, ist beibehalten worden. Stichworte sind unter anderem Wechselwirkungen mit anderen Medikamenten, Wechsel der Substanzen, Vorgehensweise bei Blutungen und die Anwendung in besonderen Situationen (u.a. Ablation, akuter Schlaganfall, KHK-Patienten mit Plättchenhemmung). Als neuestes Antikoagulans war dabei nun auch Edoxaban berücksichtigt. Der am 9. Juni online im „European Heart Journal" publizierte Update-Leitfaden ist auf der Website der ESC frei zugänglich (www.escardio.org). 